\title{
Complexity-Based Modeling of Scientific Capital: An Outline of Mathematical Theory
}

\author{
Yurij L. Katchanov and Natalia A. Shmatko \\ National Research University Higher School of Economics (HSE), 20 Myasnitskaya Ulitsa, Moscow 101000, Russia \\ Correspondence should be addressed to Yurij L. Katchanov; yurij.katchanov@gmail.com
}

Received 30 June 2014; Accepted 6 October 2014; Published 30 October 2014

Academic Editor: Sergejs Solovjovs

Copyright ( 2014 Y. L. Katchanov and N. A. Shmatko. This is an open access article distributed under the Creative Commons Attribution License, which permits unrestricted use, distribution, and reproduction in any medium, provided the original work is properly cited.

\begin{abstract}
The paper intends to contribute to a better understanding of the phenomenon of scientific capital. Scientific capital is a wellknown concept for measuring and assessing the accumulated recognition and the specific scientific power. The concept of scientific capital developed by Bourdieu is used in international social science research to explain a set of scholarly properties and practices. Mathematical modeling is applied as a lens through which the scientific capital is addressed. The principal contribution of this paper is an axiomatic characterization of scientific capital in terms of natural axioms. The application of the axiomatic method to scientific capital reveals novel insights into problem still not covered by mathematical modeling. Proposed model embraces the interrelations between separate sociological variables, providing a unified sociological view of science. Suggested microvariational principle is based upon postulate, which affirms that (under suitable conditions) the observed state of the agent in scientific field maximizes scientific capital. Its value can be roughly imagined as a volume of social differences. According to the considered macrovariational principle, the actual state of scientific field makes so-called energy functional (which is associated with the distribution of scientific capital) minimal.
\end{abstract}

\section{Introduction}

It is often very difficult to in-depth describe scientific events in sociology, using mathematical language; some data are usually unavailable or regularities are too complex. Luckily exhaustive modeling is often unnecessary, especially when a mathematical model aims to explain social structures of science rather than statistical databases on scholars and their activities. The concept of a scientific capital, SC for short, expresses a crucial social structure of present-day science. SC is an invariable property that is connected to the allocation of specific scientific power and recognition. This concept has been introduced by Bourdieu $[1,2]$. Notwithstanding achievements of the Bourdieu's approach to SC, there is no a mathematical model of SC (see, e.g., [3]).

We can only truly understand a sociological concept if we can create a mathematical model in which all of the concept's meanings and propositions are interpreted sociologically. The original formulation by Bourdieu was not mathematical. The paper aims at improving the quantitative interpretation and the logical depth of the notion of SC. The particular attention is paid to the development of a model that unambiguously expresses in mathematical language the phenomenon of SC. The proposed quantitative approach to SC makes it possible to represent meaningful amounts of SC in an additive and divisible numeric form. The suggested mathematical model of SC might be used as a conceptual framework to aid in the economic evaluation and the management tasks.

\section{Modeling of Complexity in Axiomatic Framework}

We distinguish in problems of a scientific field (SF) and SC the three basic elements listed below. These elements are what determines the real meaning of each problem.

These elements are as follows.

(1) We begin by describing the SF by introducing $d$ types of sociological quantities $X=\left(X_{1}, \ldots, X_{d}\right)$ that satisfy the defining conditions for abstract vectors. 
A scholar, or in terms of Bourdieu an agent in the SF is described only by these quantities.

(2) Quite generally, the state $s(\cdot): \Omega \rightarrow \mathbb{R}_{+}$of an agent is defined by his or her position in the SF in terms of sociological quantities $X$. For simplicity's sake, we will only consider stationary state $s(\cdot)$.

(3) The term state functional $\varphi: S \rightarrow \mathbb{R}_{+}$refers to the sociological characteristic that is uniquely defined by the state $s \in S$.

We introduce the following notation:

(i) we will use capitals $X=\left(X_{1}, \ldots, X_{d}\right)$ to denote variables and small letters $x=\left(x_{1}, \ldots, x_{d}\right)$ to denote their values;

(ii) the configuration space $\Omega$ with values $\omega=x$ is a bounded open set in the $d$-dimensional Euclidian space:

$$
\begin{aligned}
& (i=1, \ldots, d) \quad\left(\forall i: \alpha_{i}, \beta_{i} \in \mathbb{R}_{+}\right) \quad\left(\forall i: \alpha_{i}<\beta_{i}\right): \\
& \Omega=\left\{\alpha_{i}<x_{i}<\beta_{i}: x \in \mathbb{R}_{+}^{d}\right\} .
\end{aligned}
$$

Let $Z$ and $G$ be open sets in $\mathbb{R}_{+}$and $\mathbb{R}_{+}^{d} \times \mathbb{R}_{+}$, respectively. Some family of state functions

$$
\left\{s(\cdot, \zeta):\left[x_{0}(\zeta), x_{1}(\zeta)\right] \longrightarrow \mathbb{R}_{+}: \zeta \in Z\right\}
$$

is said to form a SF if the mapping $(x, \zeta) \mapsto(x, s(x, \zeta))$ is oneto-one and its image contains $G$ :

$$
\left\{(x, s): s=s(x, \zeta), x_{0}(\zeta) \leq x \leq x_{1}(\zeta), \zeta \in Z\right\} \supset G
$$

SC is a state functional $\varphi \in S^{*}$ which associates a state $s \in S$ with its value $(s, \varphi)_{S} \in \mathbb{R}_{+}$, where $(\cdot, \cdot)_{S}$ is scalar product in $S$.

From a sociological viewpoint, the reasoning of complexity can be presented in the following manner. Suppose the function $s \mapsto \Phi(s)$ is defined on the state space $S$. Let $\left\{\Phi_{\delta}\right\}_{\delta \in \Delta}$ be a family of state functions and certain state function $\Phi_{0}$ exists. It is believed that $\left\{\Phi_{\delta}\right\}_{\delta \in \Delta}$ and $\Phi_{0}$ adequately describe some states of the agent $\left\{s_{\delta}\right\}_{\delta \in \Delta} \in S, s_{0} \in S$. The following problem must be solved: how to interpret convergence of the family of states $\left\{s_{\delta}\right\}_{\delta \in \Delta}$ to the state $s_{0}$. In general, it is clear that every state function of the family of states $\left\{s_{\delta}\right\}_{\delta \in \Delta}$ must converge to the proper state function $s_{0}$ on any fixed assumption:

$$
\left\{s_{\delta}(\cdot)\right\}_{\delta \in \Delta} \longrightarrow s_{0}(\cdot) \Longrightarrow\left\{\Phi_{\delta}(\cdot)\right\}_{\delta \in \Delta} \longrightarrow \Phi_{0}(\cdot) .
$$

This principle, however, must be specified. The construction of the characteristic function of a state $s$ should be accomplished so that its convergence will condition the other state functions' convergences. Complexity, comprehended in a certain way, can serve as this function.

Under suitable assumptions, we will accept the homogeneous boundary condition $\left.s(x)\right|_{x \in \partial \Omega}=0$. The choice of this boundary condition results in the state space of the model of the agent becoming the Sobolev space $H_{0}^{1}(\Omega)=W_{0}^{1,2}\left(\Omega, \mathbb{R}_{+}^{d}\right)$. Strictly speaking, the element of the Sobolev space $H_{0}^{1}(\Omega)$ is equivalence class $[s]$ but not a concrete function $s(\cdot)$. We accept this assumption while allowing for deviation from the established rules because the elements of the Sobolev space $H_{0}^{1}(\Omega)$ are defined as concrete functions.

Furthermore, assume that in the model $M$ for each allowed state $s(\cdot) \in H_{0}^{1}(\Omega)$, the method of description $\mathrm{D}_{\mathrm{M}}(s(x))=x$ is determined. We will begin to define the complexity $\mathscr{C}_{\mathrm{D}_{\mathrm{M}}}(\cdot): \mathbb{R}^{d} \rightarrow \mathbb{R}$ of the state $s(\cdot)$ by introducing in the space $H_{0}^{1}(\Omega)$ a real-valued, monotone function $\ell(s(\cdot))$ : $H_{0}^{1}(\Omega) \rightarrow \mathbb{R}_{+}$, satisfying the following reasonable axioms (cf. [4]):

$$
\begin{aligned}
& \left(\mathrm{A}_{1}\right) \ell(s)=0 \Leftrightarrow s=0 ; \\
& \left(\mathrm{A}_{2}\right)(\forall c \in \mathbb{R}): \ell(c s)=|c| \ell(s) ; \\
& \left(\mathrm{A}_{3}\right) \ell\left(s_{1}+s_{2}\right) \leq \ell\left(s_{1}\right)+\ell\left(s_{2}\right) ; \\
& \left(\mathrm{A}_{4}\right) \ell\left(s_{1}+s_{2}\right)^{2}+\ell\left(s_{1}-s_{2}\right)^{2}=2\left(\ell\left(s_{1}\right)^{2}+\ell\left(s_{2}\right)^{2}\right) ; \\
& \left(\mathrm{A}_{5}\right)\left(\left\{s_{j}\right\}_{j=1}^{\infty} \subset H_{0}^{1}(\Omega)\right):\left\{s_{j}\right\}_{j=1}^{\infty} \downarrow 0 \Rightarrow \ell\left(\left\{s_{j}\right\}_{j=1}^{\infty}\right) \downarrow 0 .
\end{aligned}
$$

Indeed, the axioms $\left(\mathrm{A}_{1}\right)-\left(\mathrm{A}_{5}\right)$ de facto define a norm in the Sobolev space $H_{o}^{1}(\Omega)$ :

$$
\left(s(\cdot) \in H_{0}^{1}(\Omega)\right): \ell(s)=\|s\|_{H_{0}^{1}(\Omega)} .
$$

The function $\ell(s)$ will be defined as a state length $s(x)$. Evidently, the state length $\ell(s)$ depends on $D_{M}$. That is to say, essentially, that we can informally define the $\left(\mathrm{D}_{\mathrm{M}},\|\cdot\|_{H_{0}^{1}(\Omega)}\right)$ complexity $\mathscr{C}(x)$ of the state $s(x)$ as a minimum of its length (cf. [5-8]):

$$
\begin{aligned}
& \left(\mathrm{D}_{\mathrm{M}}=c_{1}\right) \quad\left(\|\cdot\|_{H_{0}^{1}(\Omega)}=c_{2}\right) \quad(x \in \bar{\Omega}): \\
& \mathscr{C}(x)=\min _{s \in H_{0}^{1}(\Omega)}\left\{\ell(s): \mathrm{D}_{\mathrm{M}}(s(x))=x\right\} .
\end{aligned}
$$

The informal basis for this definition is as follows. Let us assume that there is algorithm $\mathscr{A}$ that allows for the creation of a family of approximations $\left\{A_{k}(\cdot)\right\}_{k \in \mathscr{K}}^{\mathscr{A}}$ of the state $s(\cdot)$. Therefore, the error of approximation of the state $s(\cdot)$ is given by:

$$
\begin{aligned}
& (k \in \mathscr{K}) \quad\left(A_{k}(\cdot) \in H_{0}^{1}(\Omega)\right) \quad\left(s(\cdot) \in H_{0}^{1}(\Omega)\right): \\
& e(s(\cdot), \mathscr{A})=\sup _{k \in \mathscr{K}}\left\{\left\|s(\cdot)-A_{k}(\cdot)\right\|_{H_{0}^{1}(\Omega)}\right\} .
\end{aligned}
$$

The quantity $e(s(\cdot), \mathscr{A})$ depends on both the quality of algorithm $\mathscr{A}$ and the quality of the state $s(\cdot)$. A "simple" state is easier to approximate than a "complex" state. For statements about complexity to make sense, it is necessary to examine the sequence of the states $\left\{s_{j}(\cdot)\right\}$, with the algorithm A being fixed. If we attempt to find a form of expression (7) that is invariant to both $\left\{s_{j}(\cdot)\right\}$ and $\mathscr{A}$

$$
\widehat{e}=\inf _{s \in H_{0}^{1}(\Omega)} \inf _{\mathscr{A}}\{(s(\cdot), \mathscr{A})\},
$$

then we come to (6). The introduced axiomatic approach above is used to justify the definition (6). 
Further, assume that the state $s(x)$ satisfies the following condition:

$$
\begin{aligned}
& \left(\gamma \in \mathbb{R}_{+}\right) \quad\left(y(\cdot) \in L^{2}(\Omega)\right) \quad\left(s(\cdot) \in H_{0}^{1}(\Omega)\right): \\
& \int_{\Omega} y(x) s(x) d x=\gamma .
\end{aligned}
$$

The function $y(x): \mathbb{R}^{d} \rightarrow \mathbb{R}$ describes the external impact on an agent in the SF.

For the sake of being definite, we assume, as is usually done (see $[9$, pages 12,30$]$ ), that "energy" associated to the state $s(x)$ is given by the following formula:

$$
\begin{aligned}
& (x \in \bar{\Omega}), \\
& \left(s(\cdot) \in H_{0}^{1}(\Omega)\right), \\
& \left(0<\widehat{g} \leq g(\cdot) \in C^{1}(\bar{\Omega})\right), \\
& \left(0 \leq g_{0}(\cdot) \in C(\bar{\Omega})\right): \\
& E(s(\cdot))=\int_{\Omega}\left(g(x)|\nabla s(x)|^{2}+g_{0}(x)|s(x)|^{2}\right) d x .
\end{aligned}
$$

Note that the energy functional $E(s(\cdot))$ depends on $x$ only through the state $s$ and not explicitly. This invariance motivates the name for the functional $E(s(\cdot))[10$, pages $30,32,34]$.

In the Sobolev space $H_{o}^{1}(\Omega)$ equipped with the standard norm [11, page 59]

$$
\|s(\cdot)\|_{H_{0}^{1}(\Omega)}=\left(\int_{\Omega}\left(|\nabla s(x)|^{2}+|s(x)|^{2}\right) d x\right)^{1 / 2},
$$

we can pass to the equivalent conventional energy norm $\|s(\cdot)\|_{E}($ cf. [12, page 26]):

$$
\|s(\cdot)\|_{E}=\left(\int_{\Omega}\left(g(x)|\nabla s(x)|^{2}+g_{0}(x)|s(x)|^{2}\right) d x\right)^{1 / 2} .
$$

Taking account of (12), the definition of the complexity $\mathscr{C}(x)$ of the state $s(x)$ can be written in the form of the following axiom:

$\left(\mathrm{A}_{6}\right)$

$$
\begin{aligned}
& (x \in \bar{\Omega}) \quad\left(\gamma \in \mathbb{R}_{+}\right) \quad\left(y(\cdot) \in L^{2}(\Omega)\right): \\
& \mathscr{C}(x)=\min _{s \in H_{0}^{1}(\Omega)}\left\{\|s\|_{E}: \int_{\Omega} y(x) s(x) d x=\gamma\right\} .
\end{aligned}
$$

The state $s(x)$ problem may be formulated as a minimization of the conventional energy functional $E(s(\cdot))$ with respect to the homogeneous boundary conditions.

The following axiomatic definition of the energy functional $E(s(\cdot))$ associated to the state $s(\cdot)$ is a consequence of the using Euler's multiplier rule for the isoperimetric problem [13, pages $56-57]$ :

$$
\begin{aligned}
& (x \in \bar{\Omega}), \\
& \left(s(\cdot) \in H_{0}^{1}(\Omega)\right), \\
& \left(E(s(\cdot)): H_{0}^{1}(\Omega) \longrightarrow \mathbb{R}\right), \\
& \left(0<\widehat{g} \leq g(\cdot) \in C^{1}(\bar{\Omega})\right), \\
& \left(0 \leq g_{0}(\cdot) \in C(\bar{\Omega})\right), \\
& \left(y(\cdot) \in L^{2}(\Omega)\right): \\
& E(s(\cdot))=\int_{\Omega}\left(g(x)|\nabla s(x)|^{2}+g_{0}(x)|s(x)|^{2}\right) d x \\
& \quad+\lambda \int_{\Omega} y(x) s(x) d x \longrightarrow \min ,
\end{aligned}
$$

where $\lambda$ is the Lagrange multiplier.

The energy functional $E(s(\cdot))(14)$ associated to the state $s(\cdot)$ can be sociologically interpreted as follows:

(1) the gradient term

$$
\int_{\Omega} g(x)|\nabla s(x)|^{2} d x
$$

considers the heterogeneity of the state $s(x)$;

(2) the quadratic term

$$
\int_{\Omega} g_{0}(x)|s(x)|^{2} d x
$$

demonstrates the internal symmetry of the state $s(x)$ with respect to the norm $\|\cdot\|_{E}$, as the terms with the odd powers of $s(x)$ are absent;

(3) the term

$$
\lambda \int_{\Omega} y(x) s(x) d x
$$

with the odd power of $s(x)$, describes the continuous external impact on the agent in the SF; this term indicates the deviation of the state $s(x)$ from its "critical-point" value $s^{*}$.

It is well-known (see, e.g., [14-16]) that the functional $E(s(\cdot))$ (14) attains a minimum at a unique point $s_{*}(x) \in H_{0}^{1}(\Omega)$, which is the unique nontrivial weak solution of the SturmLiouville problem:

$$
\begin{array}{r}
\operatorname{div}\left(g(x) \nabla s_{*}(x)\right)-g_{0}(x) s_{*}(x)=\lambda y(x), \\
\left.s_{*}(x)\right|_{\partial \Omega}=0 .
\end{array}
$$

When suitable conditions are met, the weak solution matches the classical solution of $(18) s_{*}(x) \in C^{2}(\bar{\Omega})$ (cf. [17, page 294]). Therefore, extremal $s_{*}(x)$ admissible for (14) might be an ordinary (nongeneralized) function. 


\section{Complexity and Scientific Capital}

Let $T$ be a set of the scientific agents $t$, with or without indices, and consider a mapping $\Psi: T \rightarrow \Omega_{t}$ of $T$ onto the configuration space $\Omega_{t}$. The function $\Psi(\cdot)$ is the set of all pairs $\psi=(\Psi(t), t)$. This function also can be represented by the net $\left\{\omega_{t}\right\}$. Continuing this line of reasoning and taking into account [18], we see that the net $\left\{\omega_{t}\right\}$ is a social network of scholars. Obviously, the representation of the agents in SF as points in some metric space equipped with the uniform metric $\left(t, t^{\prime} \in T\right): \rho\left(\omega_{t}, \omega_{t^{\prime}}\right)$ is well-defined. It is convenient to use the normalized uniform metric distances $\varrho(\cdot, \cdot)$, such that $\forall \varrho(\cdot, \cdot) \in[0,1]$. We will say that the metric $\varrho(\cdot, \cdot)$ is the social difference (SD). The SF can be viewed as a social network of the SDs between scholars. This is natural representation of the SF since the SD can be interpreted as a relation between two scientific agents.

Further, the $t$ th agent's description involves a set

$$
(t \in T) \quad\left(t^{\prime}=1, \ldots, \bar{t}\right): \varrho_{(t)}=\left\{\varrho\left(\omega_{t}, \omega_{t^{\prime}}\right)\right\}
$$

of SDs between all corresponding pairs of agents. As a mathematical object, the set $\varrho_{(t)}$ is merely a random vector $\left(\varrho_{t}, \ldots, \varrho_{\kappa}\right), \kappa=\bar{t}(\bar{t}-1) / 2$. Moreover, we use the symbol $\varrho$ to denote a general element of the domain of the SDs and say that $\varrho$ is the independent variable.

Then we can introduce the random variable $\xi(\varrho)=\varrho$ which is the identity function of SD. The random variable $\xi$ is defined on the following probability space:

$$
\left([0,1], \mathscr{B}([0,1]), P_{\varrho}\right) \text {. }
$$

The probability density function (PDF) $f(\varrho)$ is the following set of the points. For the purpose of our study, we assume that $f(\varrho)$ is a "normal physical quantity" [19], as used in experimental science (e.g., [20, pages 543-549]). Therefore, for $f(\varrho)$, we can use methods from the classical calculus of variations without resorting to the stochastic calculus of variations $[21,22]$.

The PDF $f(\varrho)$ is an excellent candidate for a state $s(x)$ : the state of an agent may be completely specified at a given time by a certain PDF of the SD. The axiomatic definition

$\left(\mathrm{A}_{8}\right)$

$$
s(x):=f(\varrho)
$$

is the statement that we are identifying the state of the agent with the PDF of the SD.

Let us now explore the implication of our choice of $s(x)$. We can write (13) as follows:

$$
\begin{aligned}
\mathscr{C}(\varrho) & =\min _{f \in C^{2}\left([0,1], \mathbb{R}_{+}\right)}\left\{\|f\|_{E}: \int_{0}^{1} f(\varrho) d \varrho=1\right\} \\
& =\min _{f \in C^{2}\left([0,1], \mathbb{R}_{+}\right)}\left\{\sqrt{E(f(\cdot))}: \int_{0}^{1} f(\varrho) d \varrho=1\right\} .
\end{aligned}
$$

According to probability theory [23, pages 33-34], the stationary PDF $f(\varrho)$ of the family of Markov diffusion processes $\left(\xi_{t}, P_{\varrho}\right)$ in the phase space $\left(\mathbb{R}_{+}, \mathscr{B}\right)$ satisfies the stationary forward Kolmogorov equation [24] (SFKE) (which in physics is also termed the Fokker-Planck-Kolmogorov equation) with time-independent drift $a(\varrho)$ and diffusion $b(\varrho)$ coefficients. The substitution

$$
\begin{aligned}
& g(\varrho)=\exp \left(\int_{0}^{\varrho} \frac{2\left(b^{\prime}(\tau)-a(\tau)\right)}{b(\tau)} d \tau\right), \\
& g_{0}(\varrho)=\frac{\left(2 a^{\prime}(\varrho)-b^{\prime \prime}(\varrho)\right) g(\varrho)}{b(\varrho)}
\end{aligned}
$$

into (18) leads to the Cauchy problem for the following SFKE:

$$
\begin{aligned}
& \left(f(\varrho) \in C^{2}\left([0,1], \mathbb{R}_{+}\right)\right), \\
& \left(a(\varrho) \in C\left([0,1], \mathbb{R}_{+}\right)\right), \\
& \left(b(\varrho) \in C^{1}\left([0,1], \mathbb{R}_{+}\right)\right): \\
& -\frac{1}{2} \frac{d^{2}(b(\varrho) f(\varrho))}{d \varrho^{2}}+\frac{d(a(\varrho) f(\varrho))}{d \varrho}+\lambda=0, \\
& \quad f(0)=f^{0}, \quad f(1)=f^{1} .
\end{aligned}
$$

SFKE (24) cannot be satisfied for arbitrary $a(\varrho)$ and $b(\varrho)$. On the other part, if the coefficients $a(\varrho)$ and $b(\varrho)$ are constructed from the experimental data, then immediately one obtains from the SFKE (24) the following result [25, page 98]:

$$
f(\varrho)=\frac{n_{0}}{b(\varrho)} \exp \left(2 \int_{0}^{\varrho} \frac{a(r)}{b(r)} d r\right),
$$

where $n_{0}$ is a normalization constant.

According to the Lax-Milgram theorem [26, pages 41$48]$, if (24) has a weak solution $u(\varrho) \in H_{0}^{1}\left([0,1], \mathbb{R}_{+}\right)$, then this solution is unique and corresponds to the minimum of the functional $\|f\|_{E}$, and every weak solution is a classical solution $f(\varrho) \in C^{2}\left([0,1], \mathbb{R}_{+}\right)[17$, pages 292-298].

It is understood that in empirical research, the drift coefficient $a(\varrho)$ and the diffusion coefficient $b(\varrho)$ are estimated based on experimental data and correspond each time to the fixed empirical cumulative distribution function (ECDF) $F_{n}^{i}(\varrho)$. The sample $S_{N}$ from among $N$ individuals is described by a ensemble of the ECDFs $\left\{F_{n}^{(i)}\right\}_{i=1}^{N}$ extracted from the measured data. Based on the functions $\left\{F_{n}^{(i)}\right\}_{i=1}^{N}$, we construct a set of empirical probability density functions (EPDFs) $\left\{f_{i}\right\}_{i=1}^{N}$, each of which we can insert the corresponding coefficients $a_{i}$ and $b_{i}$ and the complexity $\mathscr{C}_{i}(i=1, \ldots, N)$ into. Thus, the empirically estimated complexity $\mathscr{C}_{i}$ is the minimum of the square root from the energy functional $\sqrt{E_{i}}$, that is, the minimum of the functional $\left\|f_{i}\right\|_{E}$; this statement can be reformulated in the form of the corresponding variational principle:

Among all admissible PDFs corresponding to the boundary conditions $f_{i}(0)=f_{i}^{0}, f_{i}(1)=f_{i}^{1}$ of the ith agent, the PDF, which actually describes the SD of a given agent in the SF, is assigned in 
such a way that the square root from the energy functional $\sqrt{E_{i}}$ reaches its weak local minimum (see (22)).

Thereby, we have some reason to believe that the agent in the SF volens nolens will strive to minimize energy functional $\sqrt{E_{i}}$ associated with his or her state:

$$
(i=1, \ldots, N): \sqrt{E_{i}} \longrightarrow \inf
$$

A problem that is meaningfully related to the concept of the energy functional-the problem of optimizing the distribution of the agent's the SD in the SF-can be formulated as follows. The agent will strive for the minimal value of the square root from the energy functional $\sqrt{E_{i}}$ such that

$$
(i=1, \ldots, N): \mathrm{P}\left(\mathscr{C}_{i}\right)=\inf _{f_{i}}\left\{\mathrm{P}\left(\sqrt{E_{i}}\right)\right\}
$$

where

$$
\begin{array}{r}
(i=1, \ldots, N) \quad(0<\beta=\text { const }): \\
\mathrm{P}\left(\sqrt{E_{i}}\right)=\int_{0}^{1} \exp \left(\sigma E_{i}\right) \mathrm{P}(d \varrho)
\end{array}
$$

is the probability that extremum problem (26) will not be solved for a given value of the square root from the energy functional $\sqrt{E_{i}}$ and that, instead, a constant $\sigma$ will be a scaling multiplier. That is, $\mathrm{P}\left(\sqrt{E_{i}}\right)$ plays the role of risk function; that is, it measures expected failure in the quest for recognition and scientific power. This measure indicates the probability that an agent will not earn a particular scientific income in the case of the nonoptimal distribution of his or her SD. The function $\exp \left(\beta \sqrt{E_{i}}\right)$ in (26) serves as a loss function that represents the measure of disagreement between the observed value of the square root from the energy functional and the minimal.

We turn to the minimization of $\mathrm{P}\left(\sqrt{E_{i}}\right)$ and note that

$$
-\mathrm{P}\left(\mathscr{C}_{i}\right)=\sup _{f_{i}}\left\{-\mathrm{P}\left(\sqrt{E_{i}}\right)\right\} .
$$

Regarding maximization, we should note that the functional $-\mathrm{P}\left(\sqrt{E_{i}}\right)$ can be replaced with the appropriate functional. The statements in this subsection about the energy functional $\sqrt{E_{i}}$ constitute the axiom of SC:

$\left(\mathrm{A}_{9}\right)$

$$
\mathrm{SC}_{i}=\int_{0}^{1} \exp \left(-\sigma \mathscr{C}_{i}\right) \mathrm{P}(d \varrho)
$$

There can be no conclusive reasons leading from the sociological observations to axioms $\left(A_{1}\right)-\left(A_{9}\right)$. The present axioms define SC but do not explain it. Axioms $\left(A_{1}\right)-\left(A_{9}\right)$ give a set of formal statements underlying the whole structure of the mathematical model of SC. These axioms separate the mathematical aspect from the sociological: no needs to motivate why and to explain how the concept of SC was constructed.
The functional $\mathrm{SC}_{i}$ (30) symbolizes the gain $i$ th of the agent from his or her square root from the energy functional $\sqrt{E_{i}}$, that is, the operationalization of SC. We see from definition (30) that the larger the complexity $\mathscr{C}_{i}$ is, the smaller $\mathrm{SC}_{i}$ is, although the connection between these quantities is nonlinear.

From (22), it follows that that the higher the SC, the closer the EDF of the SD is to the uniform distribution. To implement this SD distribution, the agent should be equidistant from other agents. In practice, it is possible that almost all the SDs are so large that they can be counted as approximately equal to one another.

We see that the definition of SC explicitly conforms to the definition of the square root from the energy functional. Definition (30) can be stated directly in the form of the microvariational principle, namely,

The microvariational principle affirms that the $P D F$ of the SD actually taken by given agent in the $S F$ is one for which SC is at its maximum.

The actual PDFs of the SD are those that SC have a maximum. At the same time, the actual PDF of the SD is the solution to the Cauchy problem for the SFKE (24). It is very important to emphasize that there is a complete equivalence of the abovementioned microvariational principle and corresponding SFKE. Since almost all the PDFs of the SDs can be interpreted as solutions of certain SFKEs, it is possible to claim cum grano salis that the proposed conceptual model has made, by and large, one successful prediction: it predicted the existence of SC.

\section{The Variational Principle as a Means to Define the Distribution of Scientific Capital}

We suggested that the equation for the PDF of SC can be introduced by an axiomatic approach. More specifically, we require the equation for $f(\mathrm{SC})$ to be derivable from a variational principle $d W(f, h)=0$. Here

$$
W(f(\cdot)): C^{2}\left(\left[\mathrm{SC}_{0}, \mathrm{SC}_{1}\right], \mathbb{R}_{+}\right) \longrightarrow \mathbb{R}_{+}
$$

is an unknown integral functional, and $d W(f, h)$ is its Gâteaux derivative. According to this variational principle, $d W(f, h)$ must vanish for the $\operatorname{PDF} f_{*}(\mathrm{SC})$ which describes the actual distribution of SC.

Let $G$ be an open set in the space of triples (SC, $f(\mathrm{SC})$, $\left.f^{\prime}(\mathrm{SC})\right)$, and let

$$
\begin{aligned}
\left(\mathrm{SC}, f(\mathrm{SC}), f^{\prime}(\mathrm{SC})\right) \\
\quad \longmapsto \mathscr{L}\left(\mathrm{SC}, f(\mathrm{SC}), f^{\prime}(\mathrm{SC})\right) \in C^{2}\left(G, \mathbb{R}_{+}\right) .
\end{aligned}
$$

There exist a compact set $M \subset G$ such that inclusion (SC, $\left.f(\mathrm{SC}), f^{\prime}(\mathrm{SC})\right) \in G$ holds almost everywhere if SC $\epsilon$ $\left[\mathrm{SC}_{0}, \mathrm{SC}_{1}\right]$. We consider the problem which can be reduced to the following form:

$$
\begin{aligned}
W(f(\cdot)) & =\int_{\mathrm{SC}_{0}}^{\mathrm{SC}_{1}} \mathscr{L}\left(\mathrm{SC}, f(\mathrm{SC}), f^{\prime}(\mathrm{SC})\right) d \mathrm{SC} \longrightarrow \mathrm{inf}, \\
f\left(\mathrm{SC}_{0}\right) & =f_{0}, \quad f\left(\mathrm{SC}_{1}\right)=f_{1} .
\end{aligned}
$$


If we let $f_{*}(\cdot) \in C^{2}\left(\left[\mathrm{SC}_{0}, \mathrm{SC}_{1}\right], \mathbb{R}_{+}\right)$provide the weak local minimum in problem (33) relative to the space $C^{2}\left(\left[\mathrm{SC}_{0}, \mathrm{SC}_{1}\right], \mathbb{R}_{+}\right)$, an integrant $\mathscr{L}\left(\mathrm{SC}, f(\mathrm{SC}), f^{\prime}(\mathrm{SC})\right)$ will belong to the space $C^{2}\left(\left[\mathrm{SC}_{0}, \mathrm{SC}_{1}\right] \times \mathbb{R}_{+} \times \mathbb{R}, \mathbb{R}\right)$ in the region of the extended graph:

$$
\begin{aligned}
\Gamma_{f_{*} f_{*}^{\prime}}=\{\mathrm{SC} & \in\left[\mathrm{SC}_{0}, \mathrm{SC}_{1}\right], \\
& \left.\quad f=f_{*}(\mathrm{SC}), f^{\prime}=f_{*}^{\prime}(\mathrm{SC}):\left(\mathrm{SC}, f, f^{\prime}\right)\right\} .
\end{aligned}
$$

Thereby, the first Gâteaux derivative of the functional $W(f(\cdot))$ in the neighborhood (34) on extremal $f_{*}(\cdot)$ becomes zero and yields a Euler equation, whereas the second derivative defines the quadratic functional [27]:

$$
\begin{aligned}
& \left(h \in C_{0}^{2}\left(\left[\mathrm{SC}_{0}, \mathrm{SC}_{1}\right], \mathbb{R}_{+}\right)\right): \\
& K(f(\cdot))=\int_{\mathrm{SC}_{0}}^{\mathrm{SC}_{1}}\left(P_{*}(\mathrm{SC}) h^{2}+Q_{*}(\mathrm{SC}) h^{2}\right) d \mathrm{SC} \geq 0, \\
& P_{*}(\mathrm{SC}):=\frac{1}{2} \mathscr{L}_{* f^{\prime} f^{\prime}}(\mathrm{SC}), \\
& Q_{*}(\mathrm{SC}):=\left(\frac{1}{2} \mathscr{L}_{* f f}(\mathrm{SC})-\frac{d}{d \mathrm{SC}} \mathscr{L}_{* f f^{\prime}}(\mathrm{SC})\right) .
\end{aligned}
$$

The Euler equation for the quadratic functional $K(f(\cdot))$

$$
\begin{aligned}
& \left(P_{*}(\mathrm{SC}) \in C^{1}\left(\left[\mathrm{SC}_{0}, \mathrm{SC}_{1}\right], \mathbb{R}_{+}\right)\right), \\
& \left(P_{*}(\mathrm{SC}) \geq P_{0}>0: \forall \mathrm{SC} \in\left(\mathrm{SC}_{0}, \mathrm{SC}_{1}\right)\right), \\
& \left(Q_{*}(\mathrm{SC}) \in C\left(\left[\mathrm{SC}_{0}, \mathrm{SC}_{1}\right], \mathbb{R}_{+}\right)\right): \\
& -\frac{d}{d \mathrm{SC}}\left(P_{*}(\mathrm{SC}) h^{\prime}\right)+Q_{*}(\mathrm{SC}) h=0
\end{aligned}
$$

is equal to the Jacobi equation [28] for the functional $W(f(\cdot))$. The Jacobi condition in combination with the strong Legendre condition $\left(P_{*}(\mathrm{SC})>0\right)$ results in enough conditions that the extremal $f_{*}(\cdot)$ will provide the weak local minimum for the functional $W(f(\cdot))$, which signifies the existence of a weak neighborhood:

$$
\begin{aligned}
\mathscr{O} & \left(f_{*}(\cdot), \varepsilon\right) \\
& =\left\{\left\|f(\cdot)-f_{*}(\cdot)\right\|_{C^{2}}<\varepsilon: f(\cdot) \in C^{2}\left(\left[\mathrm{SC}_{0}, \mathrm{SC}_{1}\right], \mathbb{R}_{+}\right)\right\},
\end{aligned}
$$

where the extremal $f_{*}(\cdot)$ is such that

$$
\left(\forall f(\cdot) \in \mathcal{O}\left(f_{*}(\cdot), \varepsilon\right)\right): W\left(f_{*}(\cdot)\right) \leq W(f(\cdot)) .
$$

In the weak neighborhood $\mathcal{O}\left(f_{*}(\cdot), \varepsilon\right)$, we examine an arbitrarily varied state of the $\mathrm{SF} f_{*}(\mathrm{SC})+\theta v(\mathrm{SC})$, where $\theta \in(-\varepsilon, \varepsilon)$, $\varepsilon>0, v(\mathrm{SC}) \in C_{0}^{2}\left(\left[\mathrm{SC}_{0}, \mathrm{SC}_{1}\right], \mathbb{R}\right)$, and, using (14), we calculate the state-to-state difference:

$$
\begin{aligned}
\Delta E=\left\|f_{*}(\mathrm{SC})+\theta v(\mathrm{SC})\right\|_{E}^{2}-\left\|f_{*}(\mathrm{SC})\right\|_{E}^{2} \\
=E\left(f_{*}(\mathrm{SC})+\theta v(\mathrm{SC})\right)-E\left(f_{*}(\mathrm{SC})\right) \\
=\theta\left(\int _ { \mathrm { SC } _ { 0 } } ^ { \mathrm { SC } _ { 1 } } \left(2 a(\mathrm{SC}) f_{*}(\mathrm{SC}) v(\mathrm{SC})\right.\right. \\
\left.\left.\quad-b(\mathrm{SC}) f_{*}^{\prime}(\mathrm{SC}) v^{\prime}(\mathrm{SC})\right) d \mathrm{SC}\right) \\
+\frac{\theta^{2}}{2}\left(\int _ { \mathrm { SC } _ { 0 } } ^ { \mathrm { SC } _ { 1 } } \left(2 a(\mathrm{SC})(v(\mathrm{SC}))^{2}\right.\right. \\
\left.\left.-b(\mathrm{SC})\left(v^{\prime}(\mathrm{SC})\right)^{2}\right) d \mathrm{SC}\right) .
\end{aligned}
$$

This expression can be regarded as a second-order polynomial of $\theta$, which we denote by $\pi(\theta)$. Because $f_{*}(\mathrm{SC})$ is a weak local minimizer for $W(f(\cdot))$, at $\theta=0$ the polynomial $\pi(\theta)$ has the stationary point:

$$
\begin{aligned}
\int_{\mathrm{SC}_{0}}^{\mathrm{SC}_{1}}\left(2 a(\mathrm{SC}) f_{*}(\mathrm{SC}) v(\mathrm{SC})\right. \\
\left.\quad-b(\mathrm{SC}) f_{*}^{\prime}(\mathrm{SC}) v^{\prime}(\mathrm{SC})\right) d \mathrm{SC}=0 .
\end{aligned}
$$

We examine the Sobolev space $H^{1}\left(\left(\mathrm{SC}_{0}, \mathrm{SC}_{1}\right), \mathbb{R}_{+}\right)$, which is equipped with inner product:

$$
(f, v)_{H^{1}}=(a(\mathrm{SC}) f, v)_{L^{2}}+\left(b(\mathrm{SC}) f^{\prime}, v^{\prime}\right)_{L^{2}} .
$$

The function $f(\mathrm{SC})$, which satisfies the integral identity (40) at functions

$$
\forall v(\mathrm{SC}) \in H_{0}^{1}\left(\left(\mathrm{SC}_{0}, \mathrm{SC}_{1}\right), \mathbb{R}\right),
$$

and is, in the interval $\left(\mathrm{SC}_{0}, \mathrm{SC}_{1}\right)$, a solution for the following equation:

$$
\begin{aligned}
& \left(\mathrm{SC} \in\left[\mathrm{SC}_{0}, \mathrm{SC}_{1}\right]\right): \\
& -\frac{d}{d \mathrm{SC}}\left(b(\mathrm{SC}) f^{\prime}(\mathrm{SC})\right)+2 a(\mathrm{SC}) f(\mathrm{SC})=0 .
\end{aligned}
$$

On a "physical" level of rigor, we can identify (43) with the SFKE. Consequently, $W(f(\cdot)) \equiv E(f(\cdot))$ and the variational principle

$$
\begin{aligned}
& E(f(\cdot)) \\
& \quad=\int_{\mathrm{SC}_{0}}^{\mathrm{SC}_{1}}\left(B(\mathrm{SC})\left(f^{\prime}(\mathrm{SC})\right)^{2}+A(\mathrm{SC})(f(\mathrm{SC}))^{2}\right) d \mathrm{SC} \\
& \quad \longrightarrow \inf , \\
& \quad \quad \quad\left(\mathrm{SC}_{0}\right)=f_{0}, \quad f\left(\mathrm{SC}_{1}\right)=f_{1},
\end{aligned}
$$


where

$$
\begin{aligned}
& A(\mathrm{SC})=\frac{\left(2 a^{\prime}(\mathrm{SC})-b^{\prime \prime}(\mathrm{SC})\right) B(\mathrm{SC})}{b(\mathrm{SC})} \\
& B(\mathrm{SC})=\exp \left(\int_{0}^{\mathrm{SC}} \frac{2\left(b^{\prime}(\tau)-a(\tau)\right)}{b(\tau)} d \tau\right),
\end{aligned}
$$

is a fundamental method of ascertaining the solutions to the SFKE (43).

Thereby, general formulation of the law of distribution of $\mathrm{SC}$ is the macrovariational principle.

Assuming the quasi-stationary state approximation, is characterized by a definite functional $E(f(\cdot))(44)$, the actual PDF of SC is such that takes $E(f(\cdot))$ the minimum value.

According to the principle of invariability of the phenomenological theory of SC, a function that attains the minimum energy functional (44) will be an SFKE solution (43) independent of any special integrand $\mathscr{L}\left(\mathrm{SC}, f(\mathrm{SC}), f^{\prime}(\mathrm{SC})\right)$ properties from (33).

The actual PDF of SC is an extremal of the functional $E(f(\cdot))$ (see (44)). The variational principle (44) that describes the state of the SF is located on the macrolevel of the phenomenological theory of SC, whereas the variational principle (26) that provides the PDFs of the SDs of different agents is located on the microlevel.

\section{Scientific Capital versus PageRank: An Empirical Test}

In this section, we provide a comparison between the two rankings of scholars. We compare the ranking based on the computation of SC with the ranking based on famous PageRank algorithm [29]. We limit ourselves here to a schematic case study.

We view SF as a graph, where the nodes represent scholars, and the edges between node pairs represent the SDs between nodes. The graph interpretation of the SF can be used for ranking the scientific agents. We claim that each scholar carries a scalar of "scientific success." In addition, suppose that success moves around in the graph along its edges. Rank of a scientific agent according to PageRank is given by the recursion formula:

$$
\left(\forall t, t^{\prime} \in T\right): \operatorname{Pr}(t)=\frac{1-v}{\bar{t}}+v \sum_{t^{\prime} \neq t} \frac{\operatorname{Pr}\left(t^{\prime}\right)}{W\left(t^{\prime}\right)},
$$

where $v$ is a reset parameter, $W\left(t^{\prime}\right)$ is the sum of weights of links on node $t^{\prime}$, and $\operatorname{Pr}(t)$ is the probability that the random walk is on the node $t$ and the same for $\operatorname{Pr}\left(t^{\prime}\right)$. It is readily seen that in our case we can set the following:

$$
\left(\forall t, t^{\prime} \in T\right): W\left(t^{\prime}\right)=\sum_{t^{\prime}} \varrho\left(t, t^{\prime}\right)
$$

In order to illustrate the possibility of application of the considered phenomenological theory of SC, we used the

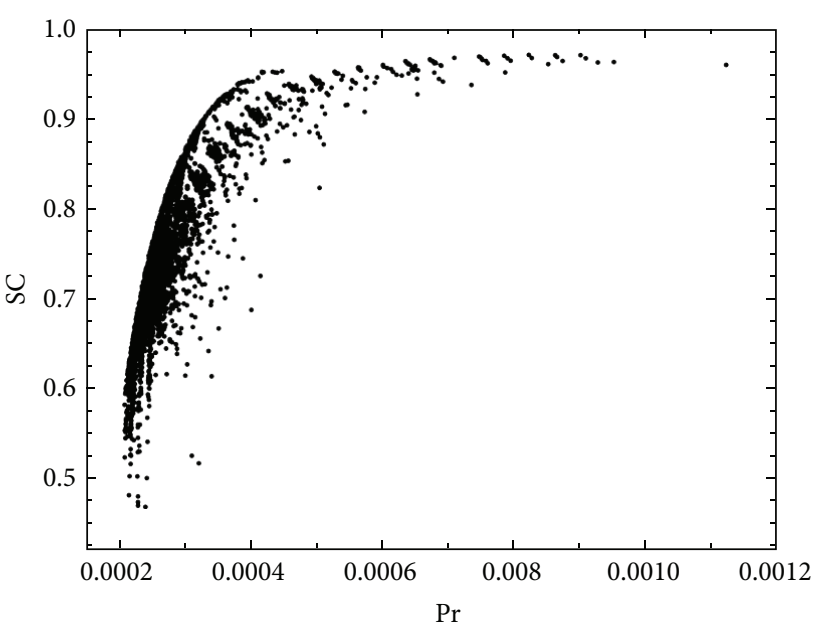

Figure 1: Dependence of SC on Pr.

data from the statistical survey "The Monitoring of the Labor Market for Highly Qualified R\&D Personnel," which was conducted in 2013 by the National Research University Higher School of Economics, Moscow. The target population included doctorate holders aged from 25 to 69 years who live and work in Russia. The sample of 3,450 respondents covers approximately $1 \%$ of the general population of all doctorate holders.

Using the indicators that Bourdieu employed in his investigation of the French SF [30], we utilize 35 variables to estimate SC of the respondents. On the other hand, we apply (46) of PageRank algorithm to the same sociological data.

The scatterplots for SC and the Pr (see Figure 1) indicate a relatively close relationship between these two sociological quantities that is nonlinear after a certain threshold. The value of Spearman's rank correlation coefficient 0.910 (the significance level $P$ is 0.000 ) corresponds to an increasing monotonic trend between SC and Pr. It is easy to see that Pr depends on the "volume" of SDs. Therefore, from the sociological point of view, this trend seems to be obvious: large Pr must show up as the bigger SC.

The above result of empirical testing can be interpreted from the practical (rather than formal statistical) standpoint, that is, admissibility of considering the variational principle to the phenomenological theory of SC.

\section{Applications}

The empirical distribution of SC might be approximated as the lognormal $\Lambda(0.753,0.132)$. The assumption that SC is distributed according to the lognormal law was checked using the Kolmogorov-Smirnov goodness-of-fit test. The value of criterion $z$ was 0.608 with a goodness of fit $\mathbf{P}$ value of 0.850 . This result can be considered appropriate for a sociological study.

In applied problems of mathematical statistics, with the help of lognormal distribution, a distribution of income 
under specific conditions is described (see, e.g., [31-34]), so an appearance of this distribution in the case of SC is an indirect confirmation of the fact that the operationalization of this term was done correctly. Thus, the distribution of SC can with some degree of reliability be qualified as lognormal that indicates the possible relevance of the microvariational principle of maximizing SC.

Since SC can be modeled as a logarithmically normal distributed random variable, then SC can be obtained as a multiplicative product of a large number of small, unrelated efficient causes; at that, the effect of each efficient cause is directly proportional to the actual value of SC [35, page 22]. Thus, we might assume SC is influenced by many random positive valued actions, which their results are independent and diminutive; these results determine the value of SC multiplicatively rather than additively. In this context, multiplicative property means that each efficient cause has some effect on SC and the result of this effect depends on the value of SC that was already reached by the time when the efficient cause had been introduced. In this scheme, the main factor is the assumption that the influence of efficient cause on the intensity of the active properties will be directly proportional to the previously achieved intensity. Despite the divisiveness of this assumption, it has long had a place in the social sciences as "the Matthew effect" [36, 37]. It follows that the sociological explanation for SC must inevitably be historical; that is, the multiplicativity of efficient causes occurs according to the historical development of SC.

A characteristic feature of the lognormal distribution of SC is the presence of large outliers, which indicates the structure-forming role of agents with small SC and agents with large SC in the Russian scientific field. In the lognormal distribution, small values for SC are inseparable from high values. Thus, the presence of the first is an inevitable price to pay for the existence of the second. In this way, the functionalization of a significant number of scholars, including those with high and low SC, is not only a sufficient reason but also a mandatory condition for the current structure of the Russian SF.

There is a correlation between SC and the age of respondents (the value of the Kendall rank-correlation coefficient $\tau$ is $0.671, \mathbf{P}=0.000)$. This result is not surprising. The first in-depth sociological study of "age, recognition, and the structure of authority in science" was performed in 1972 by Merton and Zuckerman [38]. In subsequent years, a more socially critical analysis of the age factor in the SF was realized by Bourdieu $[1,30]$. Since its theoretical introduction in 1972, a large amount of literature on the age factor in science has appeared. Thus it is quite natural that scientific recognition and administrative power increase, on a significant number of occasions, with an increase in the tenure of an agent in SF.

One might point to the gender effect on SC with a certain confidence. Spearman's rank correlation coefficient is $\rho=$ 0.723 at a statistically significant level $\mathbf{P}=0.000$. SC values for men are on average higher than for women. This kind of statistic dependence is now a commonplace of sociology of science (see, e.g., [39-46]).

\section{Conclusions}

(i) In this paper, we postulate that the PDF of the SD characterizes the state of an agent in the SF; almost all the descriptions that can be made concerning the state of an agent (under given, stationary conditions) can be deduced from a knowledge of the PDF of the SD.

(ii) The proposed approach is based on the point of view that the "complex" state of an agent in the SF requires a $\mathrm{PDF}$ (that defines the $\mathrm{SD}$ of the agent) with a long length for its description and that a "simple" state requires only a PDF with a short length. This point of view is axiomatized in the language of functional analysis. The theoretical study proved that if several assumptions that yield a collection of "natural axioms" are provided, then the complexity of the state of the agent will be computed as an estimate of the minimum value of the conventional norm of the PDF in the Hilbert-Sobolev space. The described norm corresponds to the so-called energy functional.

(iii) We identified the SC of a given agent as the quantity that is maximized by the actual PDF of the SD.

(iv) The concepts of complexity, energy, SC, and micro- or macrovariational principles provide a natural vocabulary with which we can describe significant features of the agent and the SF.

(a) Following the microvariational principle, the state of the agent in the SF is realized along admissible extremals of the square root from the energy functional, which is associated with the distribution of the SD.

(b) The microvariational principle admits a representation in the equivalent form: an actual PDF of the SD of an agent in the SF may be determined by imagining all possible PDFs that the agent could conceivably have, computing the $\mathrm{SC}$ for each of these PDFs and selecting the PDF that maximizes SC.

(c) The law of the distribution of SC may be treated more generally in the form of the macrovariational principle, whose significance is as follows: of all the conceivable laws of distribution of SC, the actually realized law is the one that yields the minimum of the energy functional.

(v) The proposed phenomenological theory of SC is organized hierarchically.

(a) On the first or microlevel are regularities, which connect disparate configurations of the SD. These regularities express the microvariational principle, according to which each agent in the SF may have his or her own microvariational principle.

(b) On the second or macrolevel are the logical relations between empirical regularities. These 
deductive relations enable us to interpret the empirical orderliness of the SD as a logical necessity. This "theoretical necessity" is reflected in the form of the macrovariational principle that determines the distribution of SC in the SF.

(vi) As a complete theory of SC, the considered theory of SC has conspicuous shortcomings. There are drift and diffusion coefficients that cannot be derived directly from the theory. The theory proposed in this paper is, nevertheless, of a phenomenological nature and does not claim to be the final theory of SC. The considered theory's general approach is that $\mathrm{SD}$ and SC are methods of describing social phenomena, not primary facts. The phenomenological theory of SC makes the consideration of the observed regularities and acquisition of their outcomes possible with the help of variational principles. The advantage of the phenomenological theory is its self-consistency and its lack of ad hoc assumptions regarding the structure of the SF, along with the absence of groundless, empirical, and special hypotheses of the scientific practices of agents.

\section{Conflict of Interests}

The authors declare that there is no conflict of interests regarding the publication of this paper.

\section{Acknowledgment}

This paper is an output of a research project implemented as part of the Basic Research Program at the National Research University Higher School of Economics (HSE).

\section{References}

[1] P. Bourdieu, "The specificity of the scientific field and the social conditions of the progress of reason," Social Science Information, vol. 14, pp. 19-47, 1975.

[2] P. Bourdieu, "The forms of capital," in Readings in Economic Sociology, N. W. Biggart, Ed., pp. 280-291, Blackwell, Malden, Mass, USA, 2002.

[3] N. K. Vitanov and M. R. Ausloos, "Knowledge epidemics and population dynamics models for describing idea diffusion," in Models of Science Dynamics: Encounters between Complexity Theory and Information Sciences, A. Scharnhorst, K. Borner, and P. V. D. Besselaar, Eds., pp. 69-125, Springer, Berlin, Germany, 2012.

[4] L. Levin, "Various measures of complexity for finite objects (axiomatic description)," Soviet Mathematics. Doklady, vol. 17, pp. 522-526, 1976.

[5] A. R. Barron and T. M. Cover, "Minimum complexity density estimation," IEEE Transactions on Information Theory, vol. 37, no. 4, pp. 1034-1054, 1991.

[6] A. Barron, J. Rissanen, and B. Yu, "The minimum description length principle in coding and modeling," IEEE Transactions on Information Theory, vol. 44, no. 6, pp. 2743-2760, 1998.
[7] P. M. Vitanyi and M. Li, "Minimum description length induction, Bayesianism, and Kolmogorov complexity," IEEE Transactions on Information Theory, vol. 46, no. 2, pp. 446-464, 2000.

[8] P. D. Grunwald, The Minimum Description Length Principle, The MIT Press, Cambridge, Mass, USA, 2007.

[9] A. Kristály, V. D. Rădulescu, and C. Varga, Variational Principles in Mathematical Physics, Geometry, and Economics: Qualitative Analysis of Nonlinear Equations and Unilateral Problems, vol. 136 of Encyclopedia of Mathematics and Its Applications, Cambridge University Press, New York, NY, USA, 2010.

[10] J. Jost and X. Li-Jost, Calculus of Variations, Cambridge University Press, Cambridge, UK, 2008.

[11] R. A. Adams and J. J. Fournier, Sobolev Spaces, Academic Press, Amsterdam, The Netherlands, 2003.

[12] V. G. Maz'ya, Sobolev Spaces: With Applications to Elliptic Partial Differential Equations, Springer, Berlin, Germany, 2011.

[13] S. G. Mikhlin, Mathematical Physics: An Advanced Course, North-Holland, Amsterdam, The Netherlands, 1970.

[14] H. Attouch, G. Buttazzo, and G. Michaille, Variational Analysis in Sobolev and BV Spaces: Applications to PDEs and Optimization, Society for Industrial and Applied Mathematics, Mathematical Programming Society, Philadelphia, Pa, USA, 2006.

[15] F. Clarke, Functional Analysis, Calculus of Variations and Optimal Control, Springer, London, UK, 2013.

[16] L. C. Evans, Partial Differential Equations, American Mathematical Society, Providence, RI, USA, 2010.

[17] H. Brézis, Functional Analysis, Sobolev Spaces and Partial Differential Equations, Springer, New York, NY, USA, 2011.

[18] M. E. Newman, "The structure and function of complex networks," SIAM Review, vol. 45, no. 2, pp. 167-256, 2003.

[19] V. N. Tutubalin, "Probability, computers, and the processing of experimental data," Physics-Uspekhi, vol. 36, no. 7, pp. 628-641, 1993.

[20] V. L. Berdichevsky, Variational Principles of Continuum Mechanics: I. Fundamentals, Springer, Berlin, Germany, 2009.

[21] K. Yasue, "Stochastic calculus of variations," Journal of Functional Analysis, vol. 41, no. 3, pp. 327-340, 1981.

[22] D. Nualart, The Malliavin Calculus and Related Topics, Springer, Berlin, Germany, 2nd edition, 2006.

[23] I. I. Gikhman and A. V. Skorokhod, The Theory of Stochastic Processes, vol. 2, Springer, Berlin, Germany, 2004.

[24] A. Kolmogoroff, "Über die analytischen Methoden in der Wahrscheinlichkeitsrechnung," Mathematische Annalen, vol. 104, no. 1, pp. 415-458, 1931.

[25] H. Risken, The Fokker-Planck Equation: Methods of Solution and Applications, Springer, Berlin, Germany, 2nd edition, 1996.

[26] M. Chipot, Elements of Nonlinear Analysis, Birkhäuser, Basel, Switzerland, 2000.

[27] W. Kratz, Quadratic Functionals in Variational Analysis and Control Theory, Akademie Verlag VCH, Berlin, Germany, 1995.

[28] A. V. Dmitruk, "The Euler-Jacobi equation in variational calculus," Mathematical Notes of the Academy of Sciences of the USSR, vol. 20, no. 6, pp. 1032-1038, 1976.

[29] S. Brin and L. Page, "The anatomy of a large-scale hypertextual web search engine," Computer Networks and ISDN Systems, vol. 30, no. 1-7, pp. 107-117, 1998.

[30] P. Bourdieu, Homo Academicus, Stanford University Press, Stanford, Calif, USA, 1988. 
[31] A. B. Atkinson and F. Bourguignon, "Income distribution and economics," in Handbook of Income Distribution, A. B. Atkinson and F. Bourguignon, Eds., vol. 1, pp. 1-58, Elsevier, Amsterdam, The Netherlands, 2000.

[32] N. L. Johnson, S. Kotz, and N. Balakrishnan, Continuous Univariate Distributions, vol. 1, Wiley-Interscience/John Wiley \& Sons, NewYork, NY, USA, 2nd edition, 1995.

[33] P. J. Lambert, The Distribution and Redistribution of Income, Manchester University Press, Manchester, UK, 3rd edition, 2001.

[34] G. S. Sahota, "Theories of personal income distribution: a survey," Journal of Economic Literature, vol. 16, pp. 1-55, 1978.

[35] J. Aitchison and J. A. C. Brown, The Lognormal Distribution, with Special Reference to Its Uses in Economics, Cambridge University Press, Cambridge, UK, 1969.

[36] R. K. Merton, “The matthew effect in science," Science, vol. 159, no. 3810, pp. 56-62, 1968.

[37] A. M. Petersen, W.-S. Jung, J.-S. Yang, and H. E. Stanley, "Quantitative and empirical demonstration of the Matthew effect in a study of career longevity," Proceedings of the National Academy of Sciences of the United States of America, vol. 108, no. 1, pp. 18-23, 2011.

[38] R. K. Merton and H. Zuckerman, "Age, aging and age structure in science," in The Sociology of Science: Theoretical and Empirical Investigations, R. K. Merton, Ed., pp. 497-559, University of Chicago Press, Chicago, Ill, USA, 2nd edition, 1979.

[39] L. Doerr, Women's Work: Gender Equality Vs. Hierarchy in the Life Sciences, Lynne Rienner Publishers, Boulder, Colo, USA, 2004.

[40] M. F. Fox, "Women and scientific careers," in Handbook of Science and Technology Studies, S. Jasanoff, Ed., pp. 205-223, Sage, Thousand Oaks, Calif, USA, 1995.

[41] S. L. Hanson, M. Schaub, and D. P. Baker, "Gender stratification in the science pipeline: a comparative analysis of seven countries," Gender \& Society, vol. 10, no. 3, pp. 271-290, 1996.

[42] S. Kulis, D. Sicotte, and S. Collins, "More than a pipeline problem: labor supply constraints and gender stratification across academic science disciplines," Research in Higher Education, vol. 43, no. 6, pp. 657-691, 2002.

[43] R. Light, "Gender stratification and publication in american science: turning the tools of science inward," Sociology Compass, vol. 3, no. 4, pp. 721-733, 2009.

[44] J. S. Long, P. D. Allison, and R. McGinnis, "Rank advancement in academic careers: sex differences and the effects of productivity," The American Sociological Review, vol. 58, no. 5, pp. 703722, 1993.

[45] M. Pezzoni, V. Sterzi, and F. Lissoni, "Career progress in centralized academic systems: social capital and institutions in France and Italy," Research Policy, vol. 41, no. 4, pp. 704-719, 2012.

[46] G. Sonnert and G. Holton, Who Succeeds in Science? The Gender Dimension, Rutgers University Press, New Brunswick, NJ, USA, 1995. 


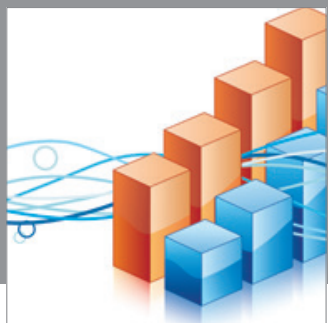

Advances in

Operations Research

mansans

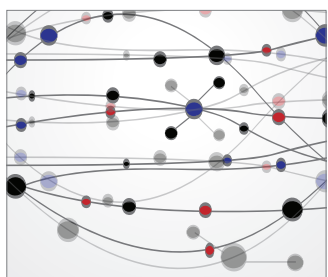

The Scientific World Journal
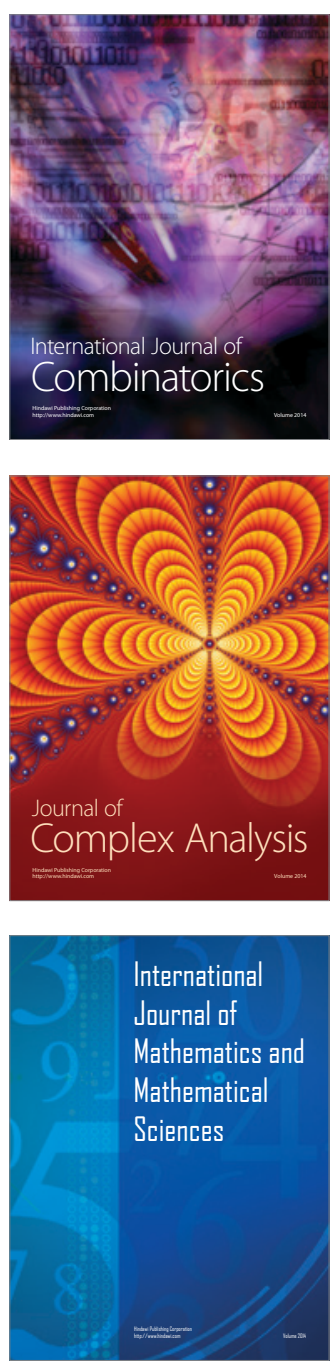
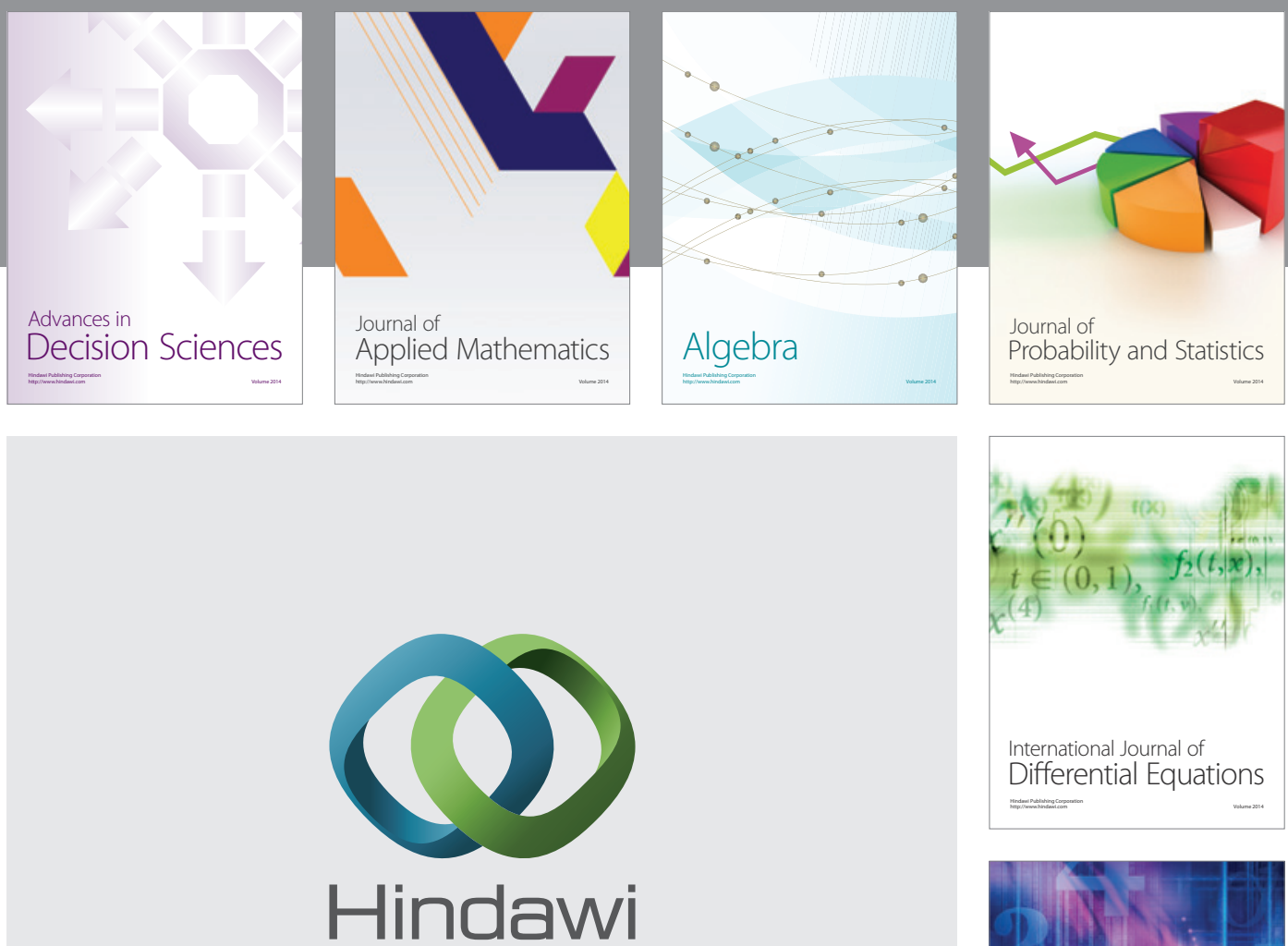

Submit your manuscripts at http://www.hindawi.com
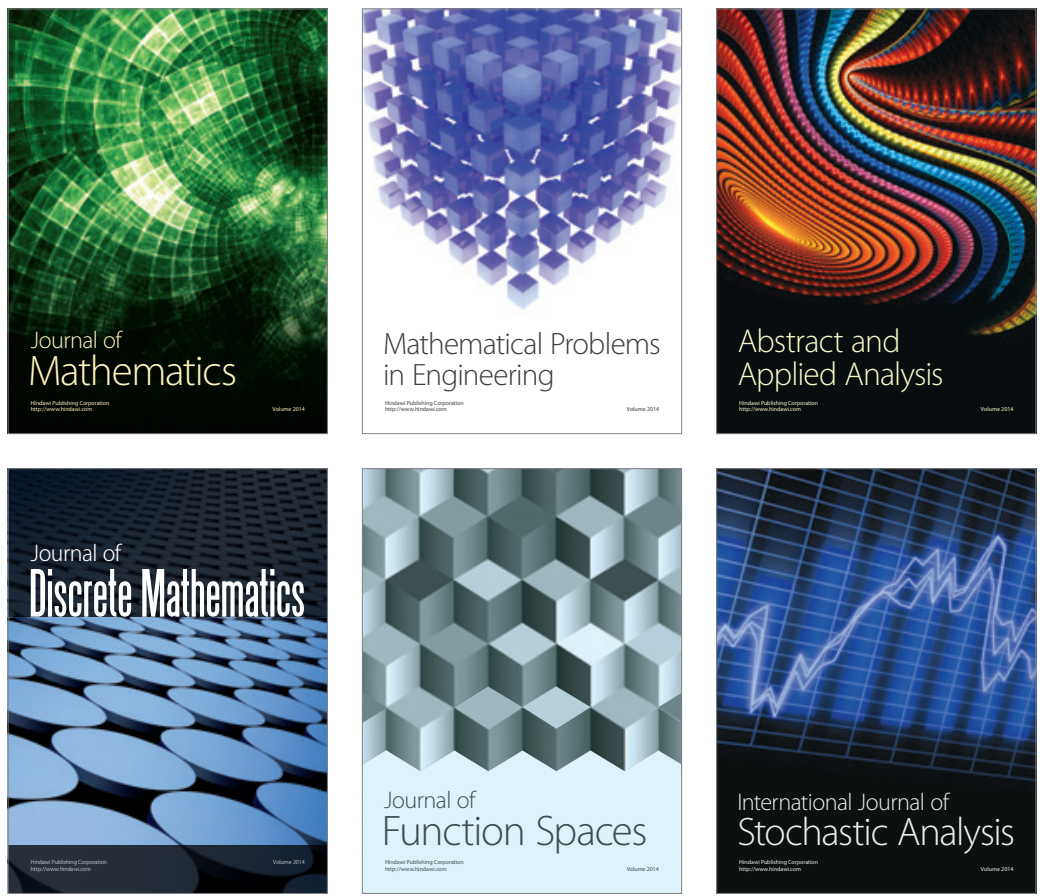

Journal of

Function Spaces

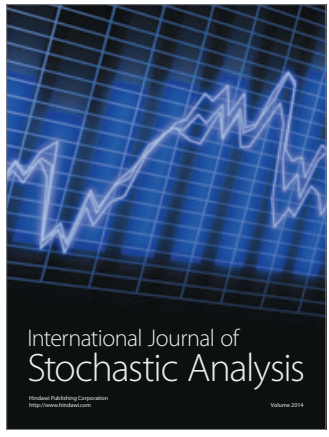

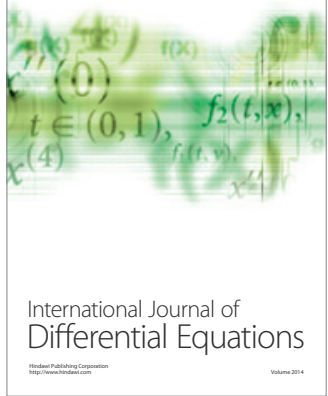
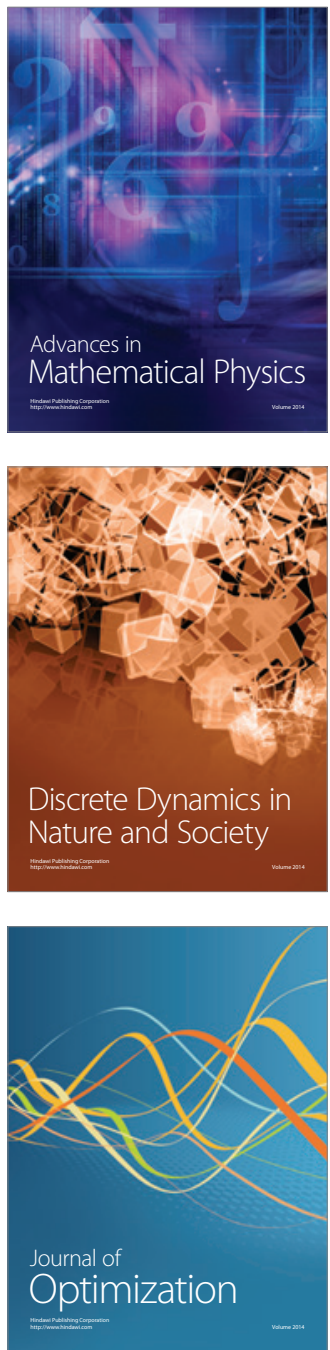